\title{
Teaching Reading Digital Texts within Neuroscientific Achievements
}

\author{
Galina V. Makotrova ${ }^{1}$, Olga A. Moiseenko ${ }^{1}$, Natalia L. Shehovskaya ${ }^{1 *}$, Elena $N$. \\ Krolevetskaya $^{1}$, and Alexander I. Korol ${ }^{2}$ \\ ${ }^{1}$ Belgorod National Research University, Belgorod, Russia \\ ${ }^{2}$ Belgorod Law Institute of Ministry of the Internal of the Russian Federation named after I. D. \\ Putilin, Belgorod, Russia
}

\begin{abstract}
The authors consider the process of teaching learners reading digital texts as pedagogical support for their search activity. Taking into account psychophysiological regularities and psychological mechanisms a teacher gets an opportunity to simulate the environment and conditions for learners to form implications, formulate questions and implement research and search for new questions based on the obtained results with the help of hypertextuality, multimedia and interactivity of digital texts in a real educational process. The purpose of the study is to identify a strategy for reading digital text. The simulation experiment for senior learners includes two opposite ways of teaching reading digital text. He/she had to start first with the short text reading, then with the corresponding picture and vice versa. The personal significance of the read text or picture, understanding of the text content and the accuracy of its reproduction were taken into account. Experimental evidence suggests the importance of using strategies for teaching reading a digital text as strategies allow the learner to act as a subject of attitude to the subject of knowledge. Teacher's digital text-based sequencing of learning situations, based on the principle of integrity, provides a sequence of future-oriented behavioral acts in the context of neuroscience achievements.
\end{abstract}

\section{Introduction}

Nowadays when choosing a text learners increasingly prefer the one consisting graphics rather than the classic verbal or traditional text [1]. Digital text consists of hypertextuality, infographics, synthesis of multimedia and verbal design [2], interactivity [3] etc. All these raise the issues of how to organize and manage the learner's activity with the digital text in off-line training on E-platforms and how to comprehend the ways of distance learning.

Teachers are concerned in decreasing the reading culture among senior teenagers when performing search tasks that involve further interpretation and evaluation of the information obtained, as stated in the international study of reading literacy PISA (Programme for International Student Assessment) [4].

\footnotetext{
* Corresponding author: kroleveckaya@,mail.ru
} 
There is a great number of questions related to the redundancy of graphic images in the text which may lead to a decreasing the speed of reading and quality of understanding the text [5], the loss of its intellectual basis [6], and the low rate of development of learner logical thinking in comparison with his/her imaginative thinking [7]. The excessive use of computer graphics possibilities in the process of interpretation of the text lead to risks of development of learners imagination, their fantasy and creativity and proves the necessity of understanding the ways of organizing the learner activity by means of the digital text by teachers [6].

Psychophysiological regularities of the human search activities are considered as his/her fundamental function that determines the integrity of the individual [8]. Knowledge of regularities allows teachers designing and realizing ways of a learner activity with the text in conditions of digital transformation of training and excludes a number of questions about necessity of replication of researches in which various ways of organizing learner training in reading digital texts are considered.

\section{Materials and methods}

In the studies of the representatives of the scientific school "Systemic Psychophysiology" $[9,10]$ it is shown that systemic psychophysiology in its methodological foundations basically overcame eclecticism and mechanistic unification of various theoretical developments due to the theory of functional systems, and significantly surpassed the neurosciences and traditional psychophysiology [9]. Systemic psychophysiology involves the study of systemogenesis, i.e., the study and modification of individual experience as a key topic in the implementation of an interdisciplinary system-evolutionary paradigm [11]. In systemic psychophysiology research is understood as a sequence of complex processes involved in the acquisition, storage and reproduction of information which results in the modification of behavior and memory is manifested as the preservation of this modification [9].

The idea of integrity highlighted within the anthropological approach takes into account the unity of biological, social and individual manifestations of a learner. Integrity allows us considering a learner as a person in the unity of his/her inner abilities and external opportunities for the implementation of his/her abilities. It also provides the understanding of the limits of what is possible according to the outcome of the learner activity under the most favorable conditions and the maximum motivation and effectiveness of the learner. At the same time, integrity focuses our attention on the importance of the "zone of proximal development" rather than on the current achievements and abilities of a learner [12] and on the understanding that the structuring, destructuring, and restructuring of a learner existing knowledge and world of life can be considered as (1) his/ her mastery of the sphere of the possible, as (2) his/her constantly renewed advance towards boundaries that separate the possible and the impossible, with variations of adaptive or destructive nature.

In the studies of the representatives of the scientific school "Systemic Psychophysiology" $[9,10]$ it is shown that systemic psychophysiology in its methodological foundations basically overcame eclecticism and mechanistic unification of various theoretical developments due to the theory of functional systems, and significantly surpassed the neurosciences and traditional psychophysiology [9]. Systemic psychophysiology involves the study of systemogenesis, i.e., the study and modification of individual experience as a key topic in the implementation of an interdisciplinary systemevolutionary paradigm [11]. In systemic psychophysiology research is understood as a sequence of complex processes involved in the acquisition, storage and reproduction of information which results in the modification of behavior and memory is manifested as the preservation of this modification [9]. 
According to the principles of systemic psychophysiology in organizing a student activity by means of a text there is a progress from the perception of the text into the change of the original text into other formats, such as questions, comics, visual notes, presentation, video sketches, supporting notes, notes in the form of quick drawings ("sketchy notes"), etc. This leads to "counter texts", new ideas in essays, compositions, created algorithms and technologies, etc. [13], i.e. in this case it is observed how the student makes sense of a written text from hypothetical knowledge ("zone of obscure knowledge") to knowledge about the lack of knowledge, and then from knowledge to new lack of knowledge. The new approach is based on the use of the basic thesis of the theory of functional systems, namely, the determinant of behavior is not the past event which is stimulus, but the future which is the result [10].

In the traditional model of teachingactivity with a text is built in type of progress of a student in learning "from lack of knowledge to knowledge" that corresponds to the methodological principle of neuroscience and traditional psychophysiology. According to the above mentioned statement, activity is caused by stimuli, and learning situations represent a linear sequence which begins with action of stimuli on receptor apparatuses and ends with response action.

The principle of systemic psychophysiology of "minimal provision of functional systems" by P.K. Anokhin is that in postnatal ontogenesis the system becomes effective for achieving positive results before all its components receive the final morphological design [14]. This principle allows teachers to present within the idea of integrity a strategy for organizing teaching learners reading digital texts. The implementation of this principle implies a continuous change of the integration process into a differentiated one initiated in the integration. N.I. Chuprikova's meta-analysis of an array of theoretical notions and facts in the field of development issues and her general universal differential-integration law of development [15], as well as earlier studies by Y.A. Ponomarev [16] show that in the process of an individual act of cognition an intuitive "emotional predestination" guiding behavior in the right direction precedes the conscious design of the solution and its verbalization.

The principle of systemic psychophysiology of "minimal provision of functional systems" by P.K. Anokhin is that in postnatal ontogenesis the organization of research among learners by means of reading digital texts assumes progress in cognition from the whole to the whole, in which the survey is realized on the basis of using multimedia objects. This process provides balancing on the borders of "I know" - "I don't know" and search for solutions as well as accumulation of new facts based on the use of hypertextuality, multimedia, interactivity, formulation of generalizations and raising new questions. 
Table 1. Images and Russian texts-analogues.

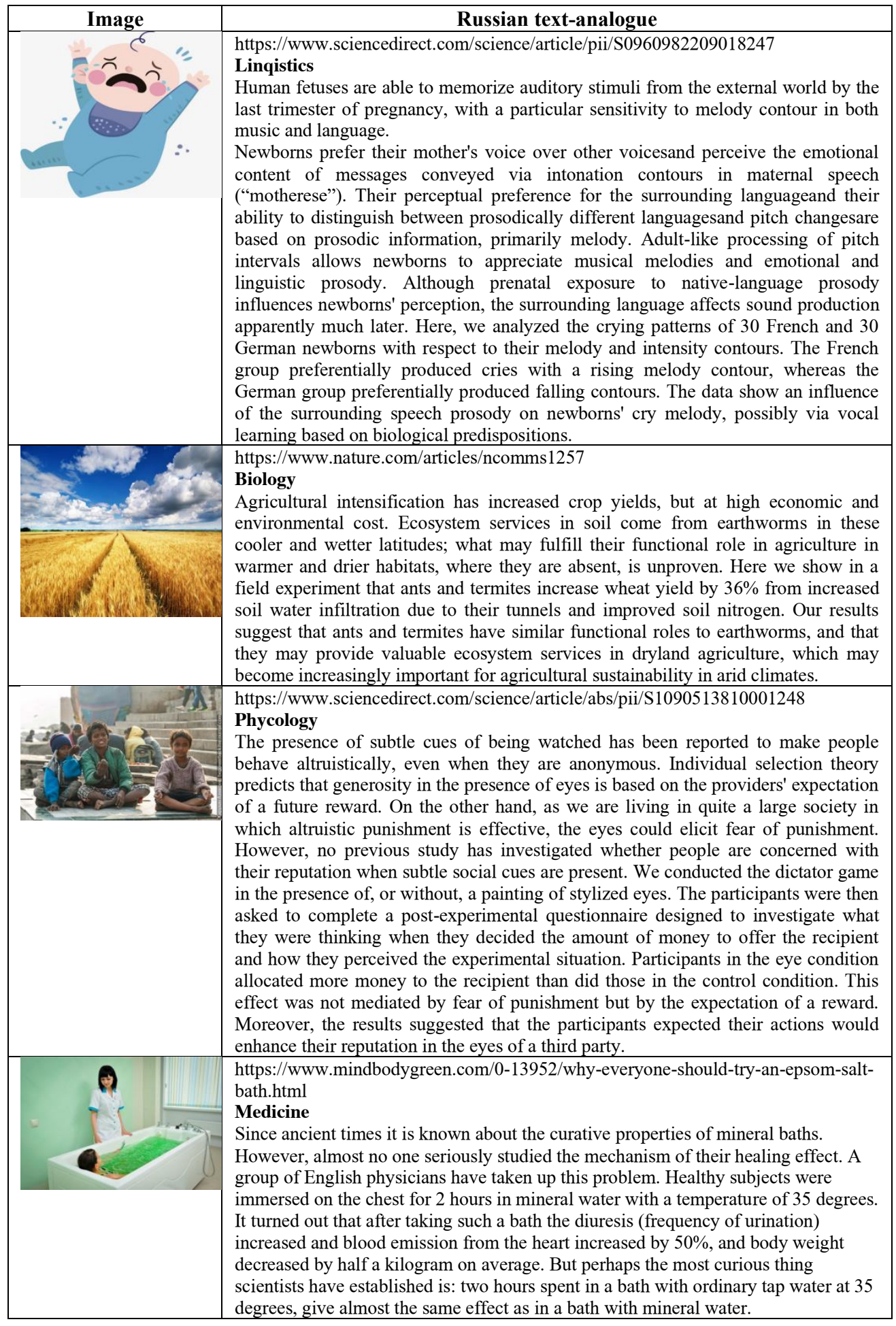


The experiment was being conducted for 22 senior learners of 10th grade which was based on the results of neuroscience research. The first stage of the experiment involved learners working on memorizing a shotr extract of a popular science article. After that learners studied pictures describing a certain image related to the content of the selected text. Chart 1 demonstates images and Russian texts-analogues as in the experiment. At the second stage of the experiment each learner first had been offered a picture and then everyone received a text for reading. After each stage of the experiment learners reproduced the read text from memory in details. When reproducing the text they were allowed using the picture.

At the third stage of the experiment learners were interviewed about the order in which it was easier for them to reproduce information.

\section{Results}

The experiment shows the following: most of learners reproduce the information and make sense of written text better in the type "from the picture to the text" which was revealed by the teacher in the course of activity is that only $29 \%$ This is probably because learners are initially attached to the image and make sense of the text based on it. Having read the text, they then look for exactly what they have discovered in the image. Besides learners spent more time on the task compared to the previous stage of working with the text. We can propose it probably associates with the processes of "thinking," analyzing and comparing the picture and the text and searching for meaning.

The survey of senior learners shows that at the first stage of the experiment they had only a memorization motivation and the image did not play a significant role. Another peculiarity of organizing learner's independent work in the type "from the picture to the text" is that only $29 \%$ of learners could understand the text correctly when working with the picture and reproduce it without mistakes. $64 \%$ of learners gave preference to memorizing text in the type "from the text to the picture". These preferences are probably connected with the typical style of work with the content in which the learner most often acts not as a subject of the attitude to the subject of knowledge but the subject of actions or activities, i.e. he/she does not realize personal meanings of cognition [17]. Organized discussion directed to the content of the picture, i.e., to the implementation of the so called "priming effect"(precedence), shows that such kind of teacher's preparatory activity provides understanding which is verified by means of creating secondary texts by learners. As a result, learners get an expression on what should be in the text. In the process of activity learners find answers to the questions in the given text and in the other digital texts. The implementation of the efficient discussion in the learning situation in which the learner makes progress in cognition through integrity is based on the principle of gradual formation of the research activity culture and three stages of development of the learners scientific abilities, such as cognitively-oriented, need-based search and dynamically active stages, identified by the psychologist N.I. Isayeva.

For research purposes the data of psychophysiologists are of great interest showing the dominance of the holistic type of thinking in the conditions of prevalence of collective life activity of people and the dominance of the analytical type of thinking which is the dominance of individual life activity of people [18]. For the research which had been conducted at school it means that such kinds of activities as selection of priority questions to the digital texts, promotion of hypotheses and criteria of estimation of results, etc. are suitable for collective forms of training. The analysis of the raised questions, search of meanings, and use of the allocated algorithms and comprehension of dynamics of one's own personal growth is characteristic for individual forms of learning. 


\section{Discussion}

Application of modern psychophysiology achievements along with the results of psychological research provides a rationale for the proposed idea of integrity in the organizing teaching learners reading digital texts. The results of a modeling experiment allowed us to designate reference points at organizing teaching learners reading digital texts in the conditions of off-line learning and prove the importance of organizing research learning through the digital text. Modeling a sequence of learning situations presenting the integrity is designed to reflect the "behavioral continuum" from the standpoint of systemic psychophysiology and the sequence of behavioral acts aimed at the future.

\section{Conclusion}

Implementation of integrity in practice according to the results of psychological and psychophysiological researches assumes consecutive change of occurrence and destruction of new cognitive connections and transitions from an emotional to rational component of learner's knowledge, from collective to individual forms of researches among learners.

In order to implement the idea of integrity in research learning teachers need to

1) use "priming effect"(precedence) as the basis for the challenge with the help of visual images in the text of a rich network of associations and the use of undifferentiated images;

2) achieve integrativeness and subject openness of the content of learning;

3) think over the complexity of search problems and collective ways of their solution.

On this basis, when organizing teaching learners reading digital texts the teacher should guide the learner from the most general holistic and not very specific to an increasingly specific, accurate and differentiated understanding of reality.

We are grateful to Belgorod National Research University for funding for this project. The research on this paper has been a thoroughly five-way collaborative matter.

\section{References}

1. Kazakova, E. I. (2016) Texts of a new nature: problems of interdisciplinary research. Psychological science and education. 21, 4,102-109.

2. Dalton B., Proctor C. P. (2008). The changing landscape of text and comprehension in the age of new literacies. Handbook of research on new literacies, 297-324.

3. Sutherland-Smith W. (2002) Web-text: Perceptions of digital reading skills in the ESL classroom. Prospect. 17, 1, 55-70.

4. Tsukerman, G.A., Kovaleva G.S., Kuznetsova, M.I. (2015). The formation of reading literacy or the new adventures of the Tian-Tolkai. Educational issues. 1, 284-300.

5. Lebedeva, M.Yu., Veselovskaya, T.S., Kupreshchenko O.F. (2020). Peculiarities of perception and understanding of digital texts: an interdisciplinary view (in Russian). Prospects of science and education. 4 (46), 94-98.

6. Serikova, T.Yu. (2010) Transformation of the concepts of "visual" and "artistic" images in contemporary culture. Izvestia Altai State University.2, 152-155.

7. Hillesund T. (2010) Digital reading spaces: How expert readers handle books, the Web and electronic paper.15.

8. Poddyakov, N.N. (2010) Mental development and self-development of the child. Moscow, Russia: Speech. 
9. Aleksandrov, Yu.I. (2004). Scientific school "Systemic psychophysiology". Psychological journal. 25, 6, 93-100.

10. Anokhin, P.K. (1974). Problem of decision making in psychology and physiology. Psychology questions. 4, 21-29.

11. Aleksandrov, Yu.I. (2012). Psychophysiological laws of science and methods of training (in Russian). 33 (6), 5-19.

12. Vygotsky L. S. (1978). Mind in society: The development of higher psychological processes. Cambridge, GB: HarvardUnivPress.

13. Oblasova, T.V. (2011). Developing the potential of schoolchildren to create secondary and counter texts. Pedagogy. 5, 52-60.

14. Anokhin, P.K. (1975). Essays on Physiology of Functional Systems. Moscow, Russia: Medicine.

15. Chuprikova, N.I.(1999). Global universal differential-integration law of development as a basis for interdisciplinary paradigm theory of development. In N.I. Chuprikova (Ed.), Development theory: Differentiation-integration paradigm (pp.7-16). Moscow, Russia.

16. Ponomarev, Ya.A. (1999). Psychology of creation. Selected psychological works. Moscow-Voronezh, Russia.

17. Perminova, L.M., Seliverstova, E.N. (2010). Didactics at the turn of epochs (XX - XXI centuries): a course of lectures for the system of higher and postgraduate teacher education. Vladimir, Russia: VSTU.

18. Kitayama, S., Uskul A.K. (2011). Culture, mind, and the brain: current evidence and future directions. Annual Review of Psychology. 62, 419- 449. 